\title{
Counting Finite-Dimensional Algebras Over Finite Field
}

\author{
Nikolaas D. Verhulst
}

\begin{abstract}
In this paper, we describe an elementary method for counting the number of non-isomorphic algebras of a fixed, finite dimension over a given finite field. We show how this method works in the case of 2dimensional algebras over the field $\mathbb{F}_{2}$.
\end{abstract}

\section{Introduction}

Classifying finite-dimensional algebras over a given field is usually a very hard problem. The first general result was a classification by Hendersson and Searle of 2-dimensional algebras over the base field $\mathbb{R}$, which appeared in 1992 ([1]). This was generalised in 2000 by Petersson ([3]), who managed to give a full classification of 2-dimensional algebras over an arbitrary base field. The methods employed in these papers are quite involved and rely on a large amount of previous work by many illustrious authors.

Our aim in this paper is to give perhaps not a classification but at least a way to compute the exact number of non-isomorphic $n$-dimensional algebras over a fixed finite field by elementary means. Indeed, nothing more complicated than linear algebra and some very basic results about group actions will be needed: we describe isomorphism classes of $n$-dimensional $K$-algebras as orbits of a certain $\mathrm{GL}_{n}(K)$-action on $\operatorname{Mat}_{n}(K)^{n}$ and use a basic result about group actions to count these orbits. In the first three sections, we give a proof based on concrete calculations, while Sect. 4 is dedicated to a more abstract alternative which avoids all computations. In Sect. 5, we work out the concrete example $n=2, K=\mathbb{F}_{2}$. 


\section{Notation and Basics}

Fix a field $K$. In this article, an algebra is understood to be a $K$-vector space $A$ equipped with a multiplication, i.e. a bilinear map $A \times A \rightarrow A$. If $a, b$ are in $A$, we will write $a b$ for the image of $(a, b)$ under this map. We do not assume algebras to have a unit or to be associative. By the dimension of an algebra we mean its dimension as a $K$-vector space. Two algebras $A$ and $A^{\prime}$ will be called isomorphic if there exists a $K$-linear bijection $f: A \rightarrow A^{\prime}$ with $f(a b)=f(a) f(b)$ for all $a, b$ in $A$. The isomorphism class of an algebra $A$ will be denoted by $[A]$. For $n \in \mathbb{N}$, we define $\operatorname{Alg}_{n}(K)$ to be the set of isomorphism classes of $n$-dimensional algebras.

Given a vector $\mathcal{M}=\left(M_{i}\right)_{i=1, \ldots, n}$ of $n(n \times n)$-matrices over $K$, we can define an algebra $\operatorname{alg}(\mathcal{M})$ which is $K^{n}$ as a $K$-vector space and for which multiplication is defined to be the unique bilinear map $K^{n} \times K^{n} \rightarrow K^{n}$ with

$$
e_{i} e_{j}=\sum_{k}\left(M_{i}\right)_{k j} e_{k}
$$

where the $e_{i}$ are the canonical basis vectors of $K^{n}$. Intuitively, this means that multiplying an element $a \in \operatorname{alg}(\mathcal{M})$ on the left with $e_{i}$ is multiplying the coordinate vector of $a$ (with respect to the canonical basis) with $M_{i}$ and interpreting the result again as a coordinate vector (with respect to the canonical basis). This allows us to define the map

$$
[\operatorname{alg}]: \operatorname{Mat}_{n}(K)^{n} \rightarrow \operatorname{Alg}_{n}(K), \mathcal{M} \mapsto[\operatorname{alg}(\mathcal{M})]
$$

which will play an important role in this paper.

Lemma 1.1. The map [alg] defined above is surjective.

Proof. Let $A$ be an $n$-dimensional algebra with basis $a_{1}, \ldots, a_{n}$. There are $\alpha_{i j, k}$ in $K$ such that $a_{i} a_{j}=\sum_{k} \alpha_{i k, j} a_{k}$ for all $1 \leq i, j \leq n$. Define the matrix $M_{i}$ by putting $\left(M_{i}\right)_{j k}=\alpha_{i j, k}$ and set $\mathcal{M}=\left(M_{i}\right)_{i=1, \ldots, n} \in \operatorname{Mat}_{n}(K)^{n}$. There is a unique linear map $\operatorname{alg}(\mathcal{M}) \rightarrow A, e_{i} \mapsto a_{i}$ which is clearly bijective and which, by construction, preserves multiplication. Hence $[A]=[\operatorname{alg}(\mathcal{M})]$.

On the other hand, [alg] is clearly not injective, since for any $\mathcal{M}=\left(M_{i}\right)_{i=1, \ldots, n}$ and $\alpha \in K^{*}$, for example, we have $[\operatorname{alg}(\mathcal{M})]=[\operatorname{alg}(\alpha \mathcal{M})]$.

\section{A Group Action on $\operatorname{Mat}_{n}(K)^{n}$}

Recall that for a given set $X$ and a group $G$ with neutral element $e$, a (right) $G$-action on $X$ is a map $\phi: X \times G \rightarrow X$ such that

(1) $\phi(x, e)=x$ for any $x \in X$,

(2) $\phi\left(x, g g^{\prime}\right)=\phi\left(\phi(x, g), g^{\prime}\right)$ for all $g, g^{\prime} \in G, x \in X$.

If $\phi$ is a $G$-action on $X$, then the $\phi$-orbit of an element $x \in X$ is the set $G(x)=\{\phi(x, g) \mid g \in G\}$. The set of $\phi$-orbits is denoted by $X / G$. The fixpoints of a $g \in G$ are the elements of $X^{g}=\{x \in X \mid \phi(x, g)=x\}$. 
Lemma 2.1. The map

$\phi: \operatorname{Mat}_{n}(K)^{n} \times \operatorname{GL}_{n}(K) \rightarrow \operatorname{Mat}_{n}(K)^{n},\left(\left[\begin{array}{c}M_{1} \\ \vdots \\ M_{n}\end{array}\right], G\right) \mapsto\left[\begin{array}{c}G^{-1} \sum_{i} G_{i 1} M_{i} G \\ \vdots \\ G^{-1} \sum_{i} G_{i n} M_{i} G\end{array}\right]$ is a $\mathrm{GL}_{n}(K)$-action on $\operatorname{Mat}_{n}(K)^{n}$.

Proof. It is clear that $\phi\left(\mathcal{M}, \mathbb{1}_{n}\right)=\mathcal{M}$ for all $\mathcal{M}$ in $\operatorname{Mat}_{n}(K)^{n}$. Take $\mathcal{M}=\left(M_{i}\right)_{i=1, \ldots, n}$ in $\operatorname{Mat}_{n}(K)^{n}$ and $G, G^{\prime}$ in $\operatorname{GL}_{n}(K)$. We have to show $\phi\left(\mathcal{M}, G G^{\prime}\right)=\phi\left(\phi(\mathcal{M}, G), G^{\prime}\right)$. The term on the right is

$$
\begin{aligned}
& \phi\left(\left[\begin{array}{c}
G^{-1} \sum_{i} G_{i 1} M_{i} G \\
\vdots \\
G^{-1} \sum_{i} G_{i n} M_{i} G
\end{array}\right], G^{\prime}\right)=\left[\begin{array}{c}
G^{\prime-1} \sum_{j} G_{j 1}^{\prime}\left(G^{-1} \sum_{i} G_{i j} M_{i} G\right) G^{\prime} \\
\vdots \\
G^{\prime-1} \sum_{j} G_{j n}^{\prime}\left(G^{-1} \sum_{i} G_{i j} M_{i} G\right) G^{\prime}
\end{array}\right] \\
& =\left[\begin{array}{c}
\left(G G^{\prime}\right)^{-1} \sum_{i}\left(G G^{\prime}\right)_{i 1} M_{i} G G^{\prime} \\
\vdots \\
\left(G G^{\prime}\right)^{-1} \sum_{i}\left(G G^{\prime}\right)_{i n} M_{i} G G^{\prime}
\end{array}\right]
\end{aligned}
$$

which is the term on the left.

Lemma 2.2. Two elements $\mathcal{M}, \mathcal{M}^{\prime}$ of $\operatorname{Mat}_{n}(K)^{n}$ are in the same $\phi$-orbit if and only if $\operatorname{alg}(\mathcal{M})$ and $\operatorname{alg}\left(\mathcal{M}^{\prime}\right)$ are isomorphic, i.e. if and only if $[\operatorname{alg}(\mathcal{M})]=$ $\left[\operatorname{alg}\left(\mathcal{M}^{\prime}\right)\right]$.

Proof. Assume $\operatorname{alg}(\mathcal{M})$ and $\operatorname{alg}\left(\mathcal{M}^{\prime}\right)$ to be isomorphic for some $\mathcal{M}=$ $\left(M_{i}\right)_{i=1, \ldots, n}$ and $\mathcal{M}^{\prime}=\left(M_{i}^{\prime}\right)_{i=1, \ldots, n}$ in $\operatorname{Mat}_{n}(K)^{n}$. Take an isomorphism $f: \operatorname{alg}(\mathcal{M}) \rightarrow \operatorname{alg}\left(\mathcal{M}^{\prime}\right)$. Since $\operatorname{alg}(\mathcal{M})$ and $\operatorname{alg}\left(\mathcal{M}^{\prime}\right)$, considered as $K$-vector spaces, are just $K^{n}$, there must be a $G \in \mathrm{GL}_{n}(K)$ such that $f(x)$ is just $G x$ for all $x \in \operatorname{alg}\left(\mathcal{M}^{\prime}\right)$. As $f$ is an isomorphism, we find

$$
G \sum_{i} x_{i} M_{i} y=f(x y)=f(x) f(y)=G x \cdot G y=\sum_{i}\left(\sum_{j} G_{i j} x_{j} M_{i}^{\prime}\right) G y
$$

for arbitrary $x, y \in \operatorname{alg}(\mathcal{M})$. In particular, if $x=e_{l}$, we find $G M_{l} y=$ $\sum_{i} G_{i l} M_{i}^{\prime} G y$ for all $y$, so $M_{l}=G^{-1} \sum_{i} G_{i l} M_{i}^{\prime} G$ showing $\phi\left(\mathcal{M}^{\prime}, G\right)=\mathcal{M}$.

Suppose now that, for given $\mathcal{M}$ and $\mathcal{M}^{\prime}$ in $\operatorname{Mat}_{n}(K)^{n}$, there is some $G \in$ $\mathrm{GL}_{n}(K)$ with $\phi\left(\mathcal{M}^{\prime}, G\right)=\mathcal{M}$. $G$ induces a function $f: K^{n} \rightarrow K^{n}, x \mapsto G x$ which is bijective as $G$ is invertible. To prove that $f$ is an isomorphism between $\operatorname{alg}(\mathcal{M})$ and $\operatorname{alg}\left(\mathcal{M}^{\prime}\right)$, it suffices to show $f\left(e_{i} e_{j}\right)=f\left(e_{i}\right) f\left(e_{j}\right)$ for all $i, j$ since $f$ is linear. We find

$$
\begin{aligned}
f\left(e_{i}\right) f\left(e_{j}\right)=\left(G e_{i}\right)\left(G e_{j}\right) & =\left(\sum_{k} G_{k i} M_{k}^{\prime}\right)\left(G e_{j}\right)=\left(\sum_{k} G_{k i} M_{k}^{\prime} G\right) e_{j} \\
& =G G^{-1}\left(\sum_{k} G_{k i} M_{k}^{\prime} G\right) e_{j}=G M_{i} e_{j}=f\left(e_{i} e_{j}\right),
\end{aligned}
$$


the penultimate equality following from $\phi\left(\mathcal{M}^{\prime}, G\right)=\mathcal{M}$.

\section{Counting Orbits}

From now on, we assume $K$ to be a finite field with $q$ elements. As a consequence of Lemma 2.2, we find that alg induces a well-defined, injective map

$$
\overline{\operatorname{alg}}: \operatorname{Mat}_{n}(K)^{n} / \operatorname{GL}_{n}(K) \rightarrow \operatorname{Alg}_{n}(K),\left(\operatorname{GL}_{n}(K)\right)(\mathcal{M}) \mapsto[\operatorname{alg}(\mathcal{M})]
$$

which is also surjective by Lemma 1.1. The number of isomorphism classes of $n$ dimensional $K$-algebras therefore equals the number of $\phi$-orbits of $\operatorname{Mat}_{n}(K)^{n}$. The following well-known result from the theory of group actions will help us count the latter:

Proposition 3.1 (Burnside's lemma). Suppose $\phi$ is an action of a finite group $G$ on a finite set $X$. Then

$$
|X / G|=\frac{1}{|G|} \sum_{g \in G}\left|X^{g}\right| .
$$

Proof. Cf. e.g. [5], p.58.

To use this lemma, we need to know the number of fixpoints of a given invertible matrix $M$. For that, we need the following definition:

Definition 3.2. For a matrix $M \in \operatorname{Mat}_{k \times l}(K)$, the vectorisation of $M$ is the $\operatorname{vector} \operatorname{vec}(M) \in K^{k l}$ obtained by stacking the columns of $M$, the first column being on top. For an element $\mathcal{M}=\left(M_{i}\right)_{i=1, \ldots, n} \in \operatorname{Mat}_{n}(K)^{n}$, we write $\operatorname{Vec}(\mathcal{M})$ for the single vector consisting of the vectorisations of all the $M_{i}$. For more on the vectorisation operation, we refer to [2].

Lemma 3.3. For an invertible matrix $M$, we have

$$
\left|\left(\operatorname{Mat}_{n}(K)^{n}\right)^{M}\right|=q^{\operatorname{dim} \operatorname{Eig}_{1}\left(M^{T} \otimes M^{T} \otimes M^{-1}\right)}
$$

where $\operatorname{Eig}_{1}(A)$ denotes the eigenspace of the matrix $A$ with eigenvalue 1.

Proof. Suppose $\mathcal{N}=\left(N_{i}\right)_{i=1, \ldots, n}$ is a fixpoint of $M$, i.e.

$$
N_{l}=M^{-1} \sum_{i} M_{i l} N_{i} M \quad \text { for all } l .
$$

It is known (see e.g. [2]) that, for arbitrary $A, B, C$ in $\operatorname{Mat}_{n}(K)$, we have $\left(B^{T} \otimes A\right) \operatorname{vec}(C)=\operatorname{vec}(A C B)$. From this, we conclude:

$$
\operatorname{Vec}\left(\left(M^{-1} N_{i} M\right)_{i=1, \ldots, n}\right)=\left(\mathbb{1}_{n} \otimes M^{T} \otimes M^{-1}\right) \operatorname{Vec}(\mathcal{N}) .
$$

We have furthermore that $\operatorname{Vec}\left(\left(\sum_{i} M_{i l} O_{i}\right)_{l=1, \ldots, n}\right)=\left(M^{T} \otimes \mathbb{1}_{n} \otimes \mathbb{1}_{n}\right) \operatorname{Vec}(\mathcal{O})$ for any $\mathcal{O}=\left(O_{i}\right)_{i=1, \ldots, n} \in \operatorname{Mat}_{n}(K)^{n}$. Consequently, $(\dagger)$ is equivalent to

$$
\operatorname{Vec}(\mathcal{N})=\left(M^{T} \otimes \mathbb{1}_{n} \otimes \mathbb{1}_{n}\right)\left(\mathbb{1}_{n} \otimes M^{T} \otimes M^{-1}\right) \operatorname{Vec}(\mathcal{N})
$$




$$
=\left(M^{T} \otimes M^{T} \otimes M^{-1}\right) \operatorname{Vec}(\mathcal{N}),
$$

so $\mathcal{N}$ is a fixpoint of $M$ if and only if $\operatorname{Vec}(\mathcal{N})$ is an eigenvector of $M^{T} \otimes M^{T} \otimes$ $M^{-1}$ with eigenvalue 1 .

Theorem 3.4. The number of non-isomorphic n-dimensional K-algebras is

$$
\left|\operatorname{Alg}_{n}(K)\right|=\frac{1}{\left|\operatorname{GL}_{n}(K)\right|} \sum_{M \in \mathrm{GL}_{n}(K)} q^{\operatorname{dim} \operatorname{Eig}_{1}\left(M^{T} \otimes M^{T} \otimes M^{-1}\right)} .
$$

Proof. By Lemma 2.2, the number of non-isomorphic $n$-dimensional $k$-algebras is the number of $\phi$-orbits. By 3.1 and 3.3, this is equal to the given formula.

\section{A Computation-Free Road to Rome}

In this section, we will outline a version of the proof which avoids all concrete computations. Grateful use has been made of an anonymous referee's report.

Suppose $A$ is a $K$-algebra. We can express a choice of basis for $A$ as a $K$-vector space isomorphism $b: K^{n} \rightarrow A$. An algebra with basis can then be seen as a pair $(A, b)$. We call two such pairs $(A, b),\left(A^{\prime}, b^{\prime}\right)$ isomorphic if there is a $K$-algebra isomorphism $f: A \rightarrow A^{\prime}$ with $f \circ b=b^{\prime}$. We denote the isomorphism class of $(A, b)$ as $\widetilde{(A, b)}$ and the set of isomorphism classes of $n$-dimensional $K$-algebras with basis as $\operatorname{AlgBas}_{n}(K)$.

For a $K$-algebra $A$, we write $\mu_{A}: A \otimes A \rightarrow A, x \otimes y \mapsto x y$. Similarly, if $M$ is an element of $\operatorname{Hom}\left(K^{n} \otimes K^{n}, K^{n}\right)$, we write $\operatorname{alg}(M)$ for the algebra which is $K^{n}$ as a $K$-vector space and with multiplication given by $x y=M(x \otimes y)$ for all $x, y$ in $K^{n}$. We can identify the set $\operatorname{AlgBas}_{n}(K)$ with $\operatorname{Hom}\left(K^{n} \otimes K^{n}, K^{n}\right)$ by the following maps

$$
\begin{aligned}
& \operatorname{AlgBas}_{n}(K) \rightarrow \operatorname{Hom}\left(K^{n} \otimes K^{n}, K^{n}\right), \quad \widetilde{(A, b)} \mapsto b^{-1} \circ \mu_{A} \circ(b \otimes b) \\
& \operatorname{Hom}\left(K^{n} \otimes K^{n}, K^{n}\right) \rightarrow \operatorname{AlgBas}_{n}(K), \quad M \mapsto(\widetilde{\operatorname{llg}(M)}, \mathrm{Id})
\end{aligned}
$$

which can be checked to be well-defined and inverse to each other.

We can define a $\mathrm{GL}_{n}(K)$-action $\phi$ on $\operatorname{AlgBas}_{n}(K)$ by $\phi((A, b), g)=(A, b \circ$ $\left.g^{-1}\right)$. The $\mathrm{GL}_{n}(K)$-orbits correspond to the fibers of the forgetful functor

$$
\operatorname{AlgBas}_{n}(K) \rightarrow \operatorname{Alg}_{n}(K),(A, b) \mapsto A,
$$

so we can count the number of non-isomorphic $n$-dimensional $K$-algebras by counting the $\mathrm{GL}_{n}(K)$-orbits of $\phi$. By the above correspondence, we get a $\mathrm{GL}_{n}(K)$-action on $\operatorname{Hom}\left(K^{n} \otimes K^{n}, K^{n}\right)$ as well and we can count the orbits of that action instead. In order to apply Burnside's lemma, we need to find the $M \in \operatorname{Hom}\left(K^{n} \otimes K^{n}, K^{n}\right)$ fixed by a given element $g \in \mathrm{GL}_{n}(K)$. These are precisely those $M$ which satisfy $g^{-1} M(g \otimes g)=M$. 
Let us recall a few results from basic linear algebra. For any two finitedimensional $K$-vector spaces $V, W$ we have, if we write $V^{*}$ for the dual space of $V, \operatorname{Hom}_{K}(V, W) \simeq V^{*} \otimes W$ via the isomorphism

$$
\Phi: V^{*} \otimes W \rightarrow \operatorname{Hom}_{K}(V, W), \phi \otimes w \mapsto(\Phi(\phi \otimes w): V \rightarrow W, v \mapsto \phi(v) w) .
$$

Similarly, a map

$$
\operatorname{Hom}_{K}(V, W) \rightarrow \operatorname{Hom}_{K}(V, W), f \mapsto a \circ f \circ b
$$

for some $a \in \operatorname{End}_{K}(W), b \in \operatorname{End}_{K}(V)$ corresponds to the map

$$
b^{*} \otimes a: V^{*} \otimes W \rightarrow V^{*} \otimes W
$$

where we have written $b^{*}$ for the dual of $b$.

In particular, we can apply this with $V=K^{n} \otimes K^{n}$ and $W=K^{n}$. Writing vec for the isomorphism $\operatorname{Hom}_{K}\left(K^{n} \otimes K^{n}, K^{n}\right) \rightarrow\left(K^{n} \otimes K^{n}\right)^{*} \otimes K^{n}$, we find that $g^{-1} M(g \otimes g)=M$ is equivalent to

$$
\left((g \otimes g)^{T} \otimes g^{-1}\right) \operatorname{vec}(M)=\operatorname{vec}(M) .
$$

We conclude that vec induces an isomorphism between the subvector space of $\operatorname{Hom}_{K}\left(K^{n} \otimes K^{n}, K^{n}\right)$ consisting of elements fixed under $g$ on the one hand and $\operatorname{Eig}_{1}\left((g \otimes g)^{T} \otimes g^{-1}\right)$ on the other hand.

\section{Example: the Case $n=2, q=2$}

Any element of $\mathrm{GL}_{2}(K)$ has, counting (algebraic) multiplicities, two eigenvalues in the algebraic closure $\bar{K}$ of $K$. Clearly, either both or none are elements of $K$ which makes counting invertible matrices with eigenvalues in $K$ considerably easier. Indeed, the only possible Jordan normal forms for a $2 \times 2$ matrix $\operatorname{are}^{1}$

$$
J_{1}=\left[\begin{array}{ll}
\alpha & \\
& \beta
\end{array}\right] \quad \text { and } \quad J_{2}=\left[\begin{array}{cc}
\alpha & 1 \\
& \alpha
\end{array}\right]
$$

for some $\alpha, \beta$ in the algebraic closure of $K$. Since $M=S J S^{-1}, M^{\prime}=S^{\prime} J^{\prime} S^{\prime-1}$ implies $M \otimes M^{\prime}=\left(S \otimes S^{\prime}\right)\left(J \otimes J^{\prime}\right)\left(S^{-1} \otimes S^{\prime-1}\right)$ and since the Jordan normal form of $M$ is also the Jordan normal form of $M^{T}$, it follows that every $M^{T} \otimes$ $M^{T} \otimes M^{-1}$ must be conjugate either to

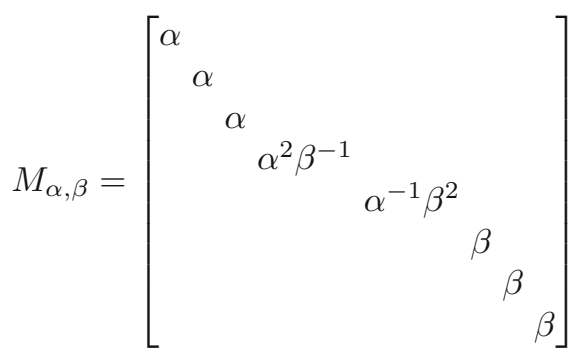

\footnotetext{
${ }^{1}$ Here and later we only write the non-zero entries in our matrices, as is usual.
} 
or to

$$
N_{\alpha}=\left[\begin{array}{ccccccc}
\alpha-1 & 1 & -\alpha^{-1} & 1 & -\alpha^{-1} & \alpha^{-1} & -\alpha^{-2} \\
\alpha & & 1 & & 1 & & \alpha^{-1} \\
& \alpha & -1 & & & 1 & -\alpha^{-1} \\
& & \alpha & & & & 1 \\
& & & \alpha & -1 & 1 & -\alpha^{-1} \\
& & & & \alpha & & 1 \\
& & & & & \alpha & -1 \\
& & & & & & \alpha
\end{array}\right]
$$

for some $\alpha, \beta \in \bar{K}$. Note that $\operatorname{dim} \operatorname{Eig}_{1}\left(N_{1}\right)=3$ unless the characteristic of $K$ is 2 , in which case $\operatorname{dim} \operatorname{Eig}_{1}\left(N_{1}\right)=4$. If $\alpha \neq 1$, we obviously have $\operatorname{dim} \operatorname{Eig}_{1}\left(N_{\alpha}\right)=$ 0 . For $M_{\alpha, \beta}$, the dimension of the eigenspace associated to 1 depends heavily on $\alpha$ and $\beta$, ranging from 8 if $\alpha=\beta=1$ to 0 if $1 \notin\left\{\alpha, \beta, \alpha^{2} \beta^{-1}, \alpha^{-1} \beta^{2}\right\}$.

We will do the computations explicitly for the concrete example of $K=$ $\mathbb{F}_{2}$. There are 6 invertible matrices, namely

$$
\mathbb{1}_{2}, \quad\left[\begin{array}{ll}
0 & 1 \\
1 & 0
\end{array}\right], \quad\left[\begin{array}{ll}
1 & 0 \\
1 & 1
\end{array}\right], \quad\left[\begin{array}{ll}
1 & 1 \\
0 & 1
\end{array}\right], \quad\left[\begin{array}{ll}
0 & 1 \\
1 & 1
\end{array}\right], \quad\left[\begin{array}{ll}
1 & 1 \\
1 & 0
\end{array}\right] .
$$

The identity obviously yields a contribution of $2^{8}$. The next three are conjugate to $J_{2}$ with $\alpha=1$, therefore yielding a contribution of $2^{4}$ each. The last two have no eigenvalues over $K$. Their eigenvalues are the roots $t_{1}, t_{2}$ of the polynomial $x^{2}+x+1$. As these roots satisfy $t_{1}^{2}=t_{2}, t_{2}^{2}=t_{1}$, both matrices give a contribution of $2^{2}$. This gives a total of $2^{8}+3 \cdot 2^{4}+2 \cdot 2^{2}=312$ which divided by the total number of invertible matrices gives $312 / 6=52$. This number fits the formulae which were obtained, using completely different methods, by Petersson and Scherer in [4].

\section{Outlook}

Theorem 3.4 suggests the following question: how many invertible $n \times n$ matrices $M$ have

$$
\operatorname{dim} \operatorname{Eig}_{1}\left(M^{T} \otimes M^{T} \otimes M^{-1}\right)=k
$$

for a given $k \in \mathbb{N}$ ? If $q$ and $n$ are fixed, this is a finite problem and can therefore be calculated, but this is rather tedious and time-consuming. Having a closed formula in $q$ and $n$ would be nice.

On the algebraic side, it would be interesting to see whether the method described in this paper can also be used to count certain subclasses of algebras, like alternating algebras, associative algebras, or division algebras. 


\section{Acknowledgements}

With the exception of Sect. 4, almost all of this work was carried out during the author's stay at the TU Dresden. The author is grateful to an anonymous referee for a very detailed and helpful report which was the basis for Sect. 4 .

Open Access. This article is licensed under a Creative Commons Attribution 4.0 International License, which permits use, sharing, adaptation, distribution and reproduction in any medium or format, as long as you give appropriate credit to the original author(s) and the source, provide a link to the Creative Commons licence, and indicate if changes were made. The images or other third party material in this article are included in the article's Creative Commons licence, unless indicated otherwise in a credit line to the material. If material is not included in the article's Creative Commons licence and your intended use is not permitted by statutory regulation or exceeds the permitted use, you will need to obtain permission directly from the copyright holder. To view a copy of this licence, visit http://creativecommons.org/ licenses/by $/ 4.0 /$.

\section{References}

[1] Althoen, S.C., Hansen, K.D.: Two-dimensional real algebras with zero divisors. Acta Sci. Math (Szeged) 56, 23-42 (1992)

[2] Henderson, H.V., Searle, S.R.: The vec-permutation matrix, the vec operator and Kronecker products: a review. Linear Multilinear Algebra 9(4), 271-288 (1981)

[3] Petersson, H.P.: The classification of two-dimensional nonassociative algebras. Results Math. 37(1-2), 120-154 (2000)

[4] Petersson, H.P., Scherer, M.: The number of nonisomorphic two-dimensional algebras over a finite field. Results Math. 42(1-2), 137-152 (2004)

[5] Rotman, J.J.: An Introduction to the Theory of Groups. Grad. Texts in Math., vol. 148. Springer, Berlin (2012). ISBN: 9781461241768

Nikolaas D. Verhulst

Delft Institute of Applied Mathematics

TU Delft

Mourik Broekmanweg 6

2628 XE Delft

The Netherlands

and

Technische Universität Dresden

Institut für Algebra

01062 Dresden

Germany

e-mail: n.d.verhulst@tudelft.nl 
Received: November 27, 2019.

Accepted: September 11, 2020.

Publisher's Note Springer Nature remains neutral with regard to jurisdictional claims in published maps and institutional affiliations. 